\title{
Meningioma consistency prediction utilizing tumor to cerebellar peduncle intensity on T2-weighted magnetic resonance imaging sequences: TCTI ratio
}

\author{
Kyle A. Smith, MD, ${ }^{1}$ John D. Leever, MD, ${ }^{2}$ Phillip D. Hylton, MD, ${ }^{1}$ Paul J. Camarata, MD, ${ }^{1}$ and \\ Roukoz B. Chamoun, MD ${ }^{1}$
}

Departments of ${ }^{1}$ Neurosurgery and ${ }^{2}$ Radiology, University of Kansas Medical Center, Kansas City, Kansas

OBJECTIVE Meningioma consistency, firmness or softness as it relates to resectability, affects the difficulty of surgery and, to some degree, the extent of resection. Preoperative knowledge of tumor consistency would affect preoperative planning and instrumentation. Several methods of prediction have been proposed, but the majority lack objectivity and reproducibility or generalizability to other surgeons. In a previous pilot study of 20 patients the authors proposed a new method of prediction based on tumor/cerebellar peduncle T2-weighted imaging intensity (TCTI) ratios in comparison with objective intraoperative findings. In the present study they sought validation of this method.

METHODS Magnetic resonance images from 100 consecutive patients undergoing craniotomy for meningioma resection were evaluated preoperatively. During surgery a consistency grade was prospectively applied to lesions by the operating surgeon, as determined by suction and/or cavitron ultrasonic surgical aspirator (CUSA) intensity. Consistency grades were A, soft; B, intermediate; and C, fibrous. Using T2-weighted MRI sequences, TCTI ratios were calculated. Analysis of the TCTI ratios and intraoperative tumor consistency was completed with ANOVA and receiver operating characteristic curves.

RESULTS Of the 100 tumors evaluated, 50 were classified as soft, 29 as intermediate, and 21 as firm. The median TCTI ratio for firm tumors was 0.88 ; for intermediate tumors, 1.5 ; and for soft tumors, 1.84 . One-way ANOVA comparing TCTI ratios for these groups was statistically significant $(p<0.0001)$. A single cutoff TCTI value of 1.41 for soft versus firm tumors was found to be $81.9 \%$ sensitive and $84.8 \%$ specific.

CONCLUSIONS The authors propose this T2-based method of tumor consistency prediction with correlation to objective intraoperative consistency. This method is quantifiable and reproducible, which expands its usability. Additionally, it places tumor consistency on a graded continuum in a clinically meaningful way that could affect preoperative surgical planning.

http://thejns.org/doi/abs/10.3171/2016.1.JNS152329

KEY WORDS meningioma; tumor consistency; ultrasonic aspirator; oncology

$\mathrm{M}$ EnINGioma consistency, its relative firmness or softness, varies along a wide continuum and affects the difficulty of surgery. ${ }^{13}$ The ability to make preoperative predictions of consistency could positively impact surgical decision making. Specifically, it would allow better tailoring of the surgical approach, available instruments, and goal resectability. ${ }^{13}$ For example, soft tumors, which are easily removed with suction and/or a low-intensity cavitron ultrasonic surgical aspi- rator (CUSA), tend to be readily debulked with quicker operative times and ease of resection. On the other hand, firmer tumors are often more difficult to dissect from critical structures, which directly impacts surgical morbidity.

Prior studies have attempted to predict tumor consistency. A variety of imaging modalities have been used for this purpose, including standard MRI sequences, proton density (PD) sequences, MR elastography, MR spectroscopy, CT, and fractional anisotropy. ${ }^{3,6,10,11}$ However, many

ABBREVIATIONS CUSA = cavitron ultrasonic surgical aspirator; $\mathrm{PD}=$ proton density; ROC = receiver operating characteristic; $\mathrm{ROI}=$ region of interest; $\mathrm{TCTI}=$ tumor to cerebellar peduncle T2-weighted imaging intensity.

SUBMITTED October 12, 2015. ACCEPTED January 7, 2016.

INCLUDE WHEN CITING Published online April 8, 2016; DOI: 10.3171/2016.1.JNS152329. 
of these techniques require special imaging equipment or training for interpretation. Fractional anisotropy values, for instance, have shown significant correlation with consistency, but this modality requires special techniques that are not widely available. ${ }^{6}$ Additionally, for most of the mentioned studies, no specific definition of "hyperintensity" or "hypointensity" exists. If one focuses on standard MRI sequences, it appears that T2-weighted sequences have the strongest correlation, whereas T1-weighted and PD sequences either lacked significant association or had very mixed results. ${ }^{1,4,13,16,19}$ Although discrepancies exist, on T2 sequences it appears that increased signal correlates with softness, and decreased signal correlates with firmness. ${ }^{3,4,6,9,10,13,15,19,20}$ Consistency is generally a characteristic rated through retrospective review of operative reports or subjective intraoperative determination.

To improve its reproducibility and generalizability, an objective method of preoperatively predicting tumor consistency was defined using intraoperative tumor consistency grading. ${ }^{14}$ This initial idea was tested in 20 patients as a pilot study to define values and investigate possible correlations. In the present study we sought validation of this method in a reasonably large patient series, utilizing tumor/cerebellar peduncle T2-weighted imaging intensity (TCTI) ratios and intraoperative classification with suction and/or ultrasonic aspiration.

\section{Methods}

\section{Patients and Study Outline}

In a small pilot series we tested a new concept for rating and predicting meningioma consistency utilizing a ratio of intensities (TCTI ratio). ${ }^{14}$ The present study serves as a statistical test for validation of this idea via consecutive imaging of 100 new patients. Institutional review board approval was obtained prior to beginning the study. Three surgeons participated in the study, and a blinded neuroradiologist (J.D.L.) reviewed preoperative MR images. Operative consistency grades and TCTI ratios were calculated independently and then correlated. Results were analyzed with 1-way ANOVA by comparing consistency groups (soft, intermediate, firm) with TCTI ratios. Additionally, receiver operating characteristic (ROC) curves were drawn to determine the optimal cutoff for the 2 extremes, soft and firm.

\section{Clinical Setting}

The surgeon used the intraoperative setting to objectively quantify the consistency of meningiomas during resection. Consistency grades were assigned based on the CUSA intensity or the use of other surgical instruments and suction (Table 1). The same CUSA machine at our institution was used for all patients. Grade A (soft) tumors could be resected with suction alone or a CUSA intensity $<40$. Grade B (intermediate) tumors required a CUSA intensity between 40 and 70 . Grade $\mathrm{C}$ (firm) tumors required higher CUSA settings $(>70)$ or other surgical adjuncts such as sharp dissection. Amplitudes listed for the CUSA represent the minimal setting necessary to adequately resect the tumor. The minimum effective CUSA setting was determined in a stepwise fashion by each sur-
TABLE 1. Grading scale for intraoperative consistency

\begin{tabular}{clc}
\hline Grade & Consistency & Method of Resection, Amplitude \\
\hline A & Soft & Suction or CUSA, $<40$ \\
\hline B & Intermediate & CUSA, $40-70$ \\
\hline C & Firm & CUSA, $>70$ \\
\hline
\end{tabular}

geon in the same manner. Higher settings could obviously be used to speed up the resection but did not figure into the consistency ranking. Furthermore, if tumors had a mixed consistency, the setting used for the majority of the tumor was the one recorded. Operating surgeons dictated either the precise minimum CUSA setting or the CUSA range (grade) in which that setting was included. In select cases in which the tumor was easily removed by suction alone, a grade of A (soft) was assigned. Tumors removed en bloc were not included in the study as neither suction nor CUSA was required for removal.

\section{Imaging}

Tumor size was recorded as the longest diameter of the tumor in relative axial, coronal, and sagittal planes. The T2-weighted MRI sequence seemed to best correlate with consistency in our previous study in which we reviewed other sequences, including T1-weighted, diffusion-weighted, and FLAIR imaging. Importantly, the T2-weighted MRI sequence was selected for its use as a standard sequence. Furthermore, T2-weighted sequences do not require special equipment or protocol or additional training for interpretation. Thus, this sequence would be more useful and versatile for places outside of tertiary care centers. The MRI protocol was as follows: TR $6000 \mathrm{msec}$, TE 101 msec, FOV $240 \mathrm{~mm}$, flip angle $150^{\circ}$, resolution matrix 320 $\times 168$, averages $(\mathrm{NEX}) 1$, and slice thickness $5 \mathrm{~mm}$.

Utilizing T2-weighted MRI sequences, we calculated TCTI ratios as described in Smith et al. (Fig. 1). ${ }^{14}$ Regions of interest (ROIs) were drawn within the tumor for an average value of intensity. The ROIs did not have preset dimensions as tumor size varies. In general, ROIs were as large as possible to get the most representative sample on the axial sequence for homogeneous tumors. For heterogeneous tumors, multiple ROIs were used until a representa-
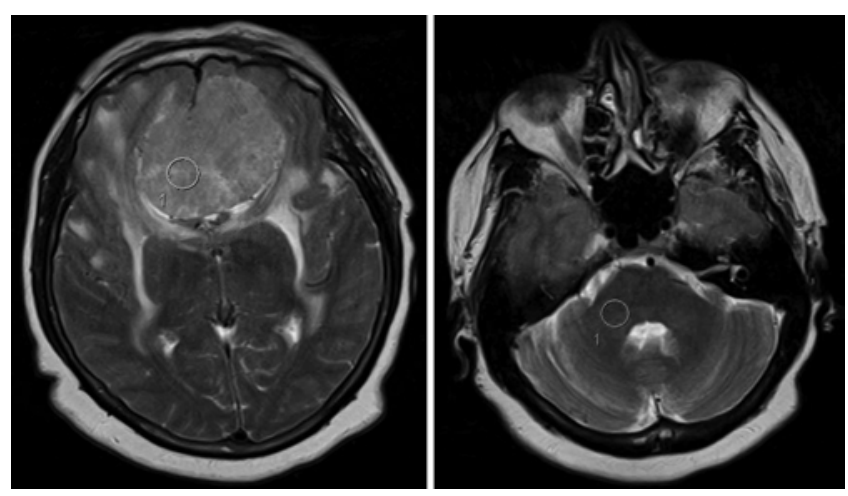

FIG. 1. Left: Tumor ROI on T2-weighted MR sequence. Right: ROI in the middle cerebellar peduncle on T2-weighted MR sequence. The TCTI ratio $=$ intensity value for $\mathrm{ROI}$ within tumor (left)/intensity value for ROI within middle cerebellar peduncle (right) $=532.7 / 266.5=1.999$. 
tive mean was determined. The middle cerebellar peduncle was chosen as an internal reference ROI, and intensity ratios were calculated. An internal reference was chosen because of the nonuniformity in MR scanners. Each machine can have intensity nonuniformity. ${ }^{17}$ In other words, the same scanner or same protocol can produce different intensities for the same tumor scanned twice. ${ }^{17}$ Furthermore, intensity is not tissue specific like Hounsfield units in CT; thus, utilizing a reference ratio to a consistent structure such as the cerebellar peduncle helps to standardize the value. ${ }^{8,12,17}$

\section{Results}

Data were collected on 100 consecutive patients. Raw data are available in Table 2. Twenty-eight patients were male; 72 were female. Patient ages ranged from 28 to 87 years, the mean age was 57.8 years, and the median age was 60 years. Tumor size ranged from 1.0 to $9.2 \mathrm{~cm}$; the mean size was $3.82 \mathrm{~cm}$, and the median was $3.6 \mathrm{~cm}$.

Fifty tumors were soft, 29 were intermediate, and 21 were firm.

Frequency distribution for the meningioma consistency groups is shown in Fig. 2. Group statistics are shown in Table 3. The TCTI ratio range for all tumors was 0.343.79 with a mean of 1.59 and median of 1.60. Grade A tumors were correlated with TCTI ratios greater than 1.63 (25th percentile). The mean TCTI ratio for this category was 1.91; the median was 1.84 ( $\mathrm{SD} \pm 0.514,95 \%$ CI 1.76 2.05). Grade B tumors were correlated with TCTI ratios of 1.33-1.63. The mean TCTI ratio was 1.49; the median was $1.5(\mathrm{SD} \pm 0.289,95 \% \mathrm{CI} 1.38-1.60)$. Grade $\mathrm{C}$ tumors were correlated with TCTI ratios less than 1.27 (75th percentile). The mean TCTI ratio was 1.01; the median was $0.88(\mathrm{SD} \pm 0.419,95 \%$ CI $0.82-1.20)$.

Comparison of consistency grades and TCTI ratios was done with 1-way ANOVA. The TCTI ratios by each grade were statistically significant $(\mathrm{p}<0.0001)$. The TCTI ratios correlated with intraoperative consistency ratings.

Meningioma consistency follows a continuum, and there is overlap of the intermediate group with soft and firm consistencies, but clinical relevance lies with identification of the extremes (soft vs firm) or those that probably do not require surgical adjuncts versus those that are extremely difficult to resect. An ROC curve was completed

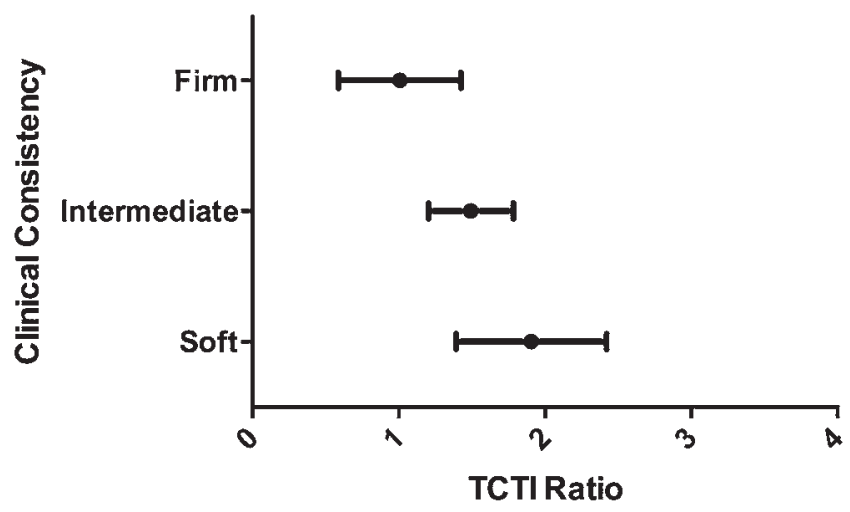

FIG. 2. Frequency distribution for TCTI ratios by tumor consistency. for these consistency extremes to determine a single number that could be used to quickly identify the consistency of the tumor. The optimal cutoff value was determined to be a TCTI ratio of 1.41 , with soft tumors $\geq 1.41$ and firm tumors $<1.41$. This TCTI ratio cutoff is $81.9 \%$ sensitive and $84.8 \%$ specific.

Figure 3 shows CUSA settings versus TCTI ratios.

\section{Discussion}

As surgery is the mainstay of treatment for meningiomas, tumor consistency is an important characteristic, as are tumor location and size. The difficulty of surgery and resectability is generally dependent on tumor consistency, ${ }^{4,6,13}$ For instance, when tumors originate from the skull base or encase neurovascular structures, a firm consistency makes the surgery rather painstaking and higher risk. The outcome is directly dependent on these characteristics. Furthermore, a firm consistency also contributes to increased operative time and may help to predict a surgeon's case scheduling.

No single objective and reproducible method of predicting tumor consistency currently exists. A variety of imaging sequences and modalities are discussed in the literature with no clear consensus and, at times, conflicting results (Table 4). Imaging modalities used include MRI, CT, MR elastography, MR spectroscopy, and fractional anisotropy. Using conventional MRI, many authors have performed studies on the correlation of MR intensity and meningioma consistency. Hoover et al., Suzuki et al., and Sitthinamsuwan et al. demonstrated a relationship between tumor consistency and T2 intensity.,13,16 Yamaguchi et al. similarly demonstrated the correlation of T2 and PD hyperintensity with a softer consistency. ${ }^{19}$ Other reports have also shown a correlation between $\mathrm{T} 2$ intensity and tumor consistency. ${ }^{2,18,21}$ In a relatively recent study, Maiuri et al. compared the $\mathrm{T} 2$ intensity of tumor to cortex and found

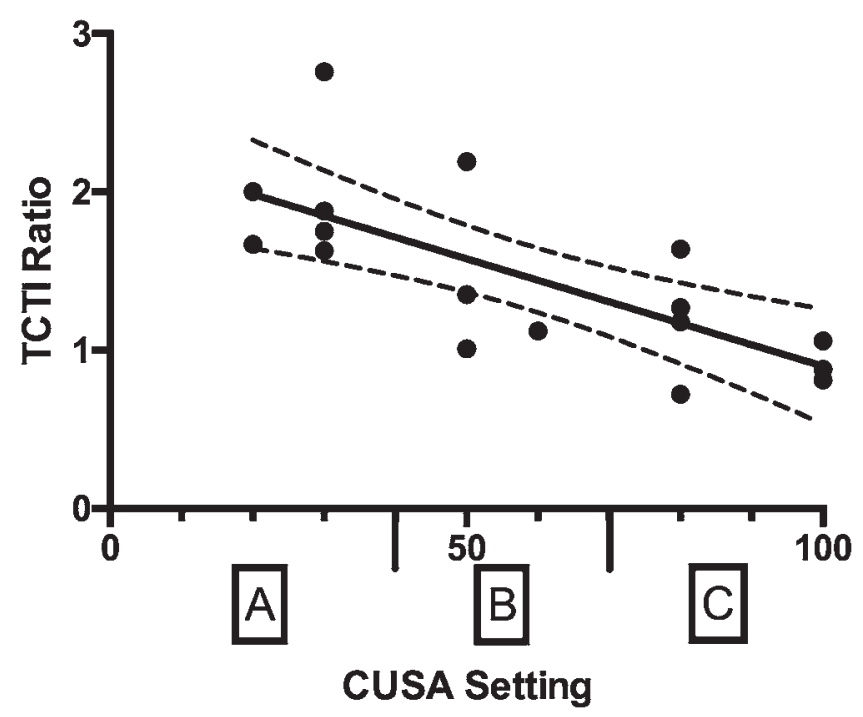

FIG. 3. Graph of CUSA settings versus TCTI ratios. Tumor consistency Grades A, B, and C are listed below the CUSA intensity (x-axis). The linear regression of the mean is denoted by the solid line. The dotted lines represent the $95 \%$ confidence interval. 
TABLE 2. Summary of data on 100 patients with meningioma

\begin{tabular}{|c|c|c|c|c|c|c|c|}
\hline Case No. & Age (yrs) & Sex & Tumor Location & Size (cm) & TCTI Ratio & Clinical Consistency & CUSA Setting \\
\hline 1 & 68 & M & Sphenoid wing & 4.0 & 1.96 & Soft & $0-40$ \\
\hline 2 & 71 & M & Olfactory groove & 3.3 & 2.8 & Soft & $0-40$ \\
\hline 3 & 54 & $\mathrm{~F}$ & Sellar-suprasellar & 2.3 & 1.66 & Soft & $0-40$ \\
\hline 4 & 45 & $\mathrm{~F}$ & Lt clinoid & 3.6 & 1.88 & Soft & $0-40$ \\
\hline 5 & 49 & $\mathrm{~F}$ & Sphenoid wing & 5.6 & 1.79 & Soft & $0-40$ \\
\hline 6 & 66 & $\mathrm{~F}$ & Planum sphenoidale & 2.7 & 2.16 & Soft & $0-40$ \\
\hline 7 & 58 & M & Tuberculum sella & 7.5 & 2.53 & Soft & $0-40$ \\
\hline 8 & 61 & M & Foramen magnum & 4.9 & 1.51 & Soft & $0-40$ \\
\hline 9 & 40 & $\mathrm{~F}$ & Petroclival & 4.7 & 1.86 & Soft & $0-40$ \\
\hline 10 & 57 & $\mathrm{~F}$ & Lt clinoid & 3.5 & 1.56 & Soft & $0-40$ \\
\hline 11 & 81 & M & Rt frontal convexity & 3.7 & 3.79 & Soft & $0-40$ \\
\hline 12 & 67 & M & Parasagittal & 9.2 & 1.63 & Soft & 30 \\
\hline 13 & 38 & $\mathrm{~F}$ & Intraventricular & 1.3 & 1.24 & Soft & $0-40$ \\
\hline 14 & 49 & M & Rt frontal convexity & 5.3 & 2.19 & Soft & $0-40$ \\
\hline 15 & 15 & $\mathrm{~F}$ & Lt periclinoid & 4.9 & 1.63 & Soft & $0-40$ \\
\hline 16 & 60 & $\mathrm{~F}$ & Planum sphenoidale & 6.5 & 1.85 & Soft & $0-40$ \\
\hline 17 & 67 & $\mathrm{~F}$ & Planum sphenoidale & 5.0 & 1.81 & Soft & $0-40$ \\
\hline 18 & 64 & $\mathrm{~F}$ & Rt falcine & 2.9 & 2.49 & Soft & $0-40$ \\
\hline 19 & 68 & $\mathrm{~F}$ & Olfactory groove & 6.2 & 1.52 & Soft & $0-40$ \\
\hline 20 & 61 & $\mathrm{~F}$ & Tuberculum sella & 3.0 & 1.88 & Soft & $0-40$ \\
\hline 21 & 54 & M & Lt frontal & 4.3 & 1.68 & Soft & $0-40$ \\
\hline 22 & 47 & $\mathrm{~F}$ & Lt CPA & 2.8 & 1.90 & Soft & $0-40$ \\
\hline 23 & 64 & $\mathrm{~F}$ & Intraventricular & 4.9 & 1.83 & Soft & $0-40$ \\
\hline 24 & 61 & M & Lt periclinoid & 1.9 & 1.69 & Soft & $0-40$ \\
\hline 25 & 52 & $\mathrm{~F}$ & Lt falcine & 6.3 & 1.54 & Soft & $0-40$ \\
\hline 26 & 60 & $\mathrm{~F}$ & Tuberculum sella & 2.0 & 1.41 & Soft & $0-40$ \\
\hline 27 & 55 & $\mathrm{~F}$ & Foramen magnum & 3.0 & 1.85 & Soft & $0-40$ \\
\hline 28 & 47 & M & Rt frontal convexity & 4.8 & 1.71 & Soft & $0-40$ \\
\hline 29 & 55 & M & Olfactory groove & 4.1 & 1.80 & Soft & $0-40$ \\
\hline 30 & 36 & $\mathrm{~F}$ & Planum sphenoidale & 3.6 & 1.75 & Soft & 30 \\
\hline 31 & 47 & $\mathrm{~F}$ & Rt frontal convexity & 3.0 & 1.88 & Soft & 30 \\
\hline 32 & 65 & $\mathrm{~F}$ & Rt CPA & 2.3 & 2.22 & Soft & $0-40$ \\
\hline 33 & 64 & $\mathrm{~F}$ & Cervicothoracic & 3.0 & 0.36 & Soft & $0-40$ \\
\hline 34 & 34 & $\mathrm{~F}$ & Tuberculum sella & 1.0 & 1.96 & Soft & $0-40$ \\
\hline 35 & 68 & $\mathrm{~F}$ & Lt posterior fossa & 3.1 & 1.95 & Soft & $0-40$ \\
\hline 36 & 37 & $\mathrm{~F}$ & Olfactory groove & 5.0 & 1.67 & Soft & 20 \\
\hline 37 & 80 & $\mathrm{~F}$ & Rt CPA & 3.7 & 1.52 & Soft & $0-40$ \\
\hline 38 & 47 & $\mathrm{~F}$ & Rt sphenoid wing & 3.9 & 2.11 & Soft & $0-40$ \\
\hline 39 & 70 & M & Rt parieto-occipital & 2.7 & 2.88 & Soft & $0-40$ \\
\hline 40 & 76 & $\mathrm{~F}$ & Sella & 4.0 & 1.60 & Soft & $0-40$ \\
\hline 41 & 64 & $\mathrm{~F}$ & Falcine & 4.7 & 2.42 & Soft & $0-40$ \\
\hline 42 & 56 & $\mathrm{~F}$ & Rt sphenoid wing & 3.2 & 1.72 & Soft & $0-40$ \\
\hline 43 & 71 & M & Rt sphenoid wing & 1.9 & 2.57 & Soft & $0-40$ \\
\hline 44 & 42 & $\mathrm{~F}$ & Lt sphenoid wing & 1.9 & 2.25 & Soft & $0-40$ \\
\hline 45 & 40 & M & Posterior fossa & 4.4 & 2.05 & Soft & $0-40$ \\
\hline 46 & 31 & M & Rt parietal & 2.5 & 2.76 & Soft & 30 \\
\hline 47 & 64 & M & Lt temporal & 3.9 & 1.81 & Soft & $0-40$ \\
\hline 48 & 72 & $\mathrm{~F}$ & Olfactory groove & 5.8 & 2.00 & Soft & 20 \\
\hline 49 & 61 & $\mathrm{~F}$ & Rt petrous apex & 1.6 & 1.25 & Soft & $0-40$ \\
\hline
\end{tabular}


» CONTINUED FROM PAGE 245

TABLE 2. Summary of data on 100 patients with meningioma

\begin{tabular}{|c|c|c|c|c|c|c|c|}
\hline Case No. & Age (yrs) & Sex & Tumor Location & Size $(\mathrm{cm})$ & TCTI Ratio & Clinical Consistency & CUSA Setting \\
\hline 50 & 73 & $\mathrm{~F}$ & Rt middle fossa & 3.3 & 1.44 & Soft & $0-40$ \\
\hline 51 & 50 & $\mathrm{~F}$ & Rt parietal convexity & 4.7 & 1.74 & Intermediate & $>40$ to $<70$ \\
\hline 52 & 64 & $\mathrm{M}$ & Sphenoid wing & 4.1 & 1.27 & Intermediate & $>40$ to $<70$ \\
\hline 53 & 47 & $\mathrm{~F}$ & Lt parietal convexity & 3.5 & 1.38 & Intermediate & $>40$ to $<70$ \\
\hline 54 & 68 & $\mathrm{~F}$ & Lt clinoid & 5.9 & 1.13 & Intermediate & $>40$ to $<70$ \\
\hline 55 & 36 & M & $\begin{array}{l}\text { Lt anterior fossa/cribri- } \\
\text { form plate }\end{array}$ & 2.8 & 1.44 & Intermediate & $>40$ to $<70$ \\
\hline 56 & 41 & $\mathrm{~F}$ & Tuberculum sella & 4.0 & 1.72 & Intermediate & $>40$ to $<70$ \\
\hline 57 & 76 & $\mathrm{~F}$ & Rt anterior fossa & 1.8 & 1.64 & Intermediate & $>40$ to $<70$ \\
\hline 58 & 61 & $\mathrm{~F}$ & Lt frontal convexity & 3.4 & 1.6 & Intermediate & $>40$ to $<70$ \\
\hline 59 & 79 & $\mathrm{M}$ & Tuberculum sella & 3.3 & 1.75 & Intermediate & $>40$ to $<70$ \\
\hline 60 & 69 & $\mathrm{~F}$ & Rt frontal convexity & 3.3 & 1.01 & Intermediate & $>40$ to $<70$ \\
\hline 61 & 47 & $\mathrm{~F}$ & Lt occipitotemporal & 3.4 & 1.35 & Intermediate & 50 \\
\hline 62 & 57 & $\mathrm{~F}$ & Rt posterior fossa & 4.1 & 1.46 & Intermediate & $>40$ to $<70$ \\
\hline 63 & 48 & $\mathrm{~F}$ & Pineal region & 4.8 & 1.64 & Intermediate & $>40$ to $<70$ \\
\hline 64 & 63 & $\mathrm{M}$ & Rt falcine & 8.5 & 1.40 & Intermediate & $>40$ to $<70$ \\
\hline 65 & 63 & $\mathrm{~F}$ & Foramen magnum & 1.8 & 1.46 & Intermediate & $>40$ to $<70$ \\
\hline 66 & 60 & $\mathrm{~F}$ & Sphenoid wing & 3.1 & 2.19 & Intermediate & 50 \\
\hline 67 & 72 & $\mathrm{~F}$ & Rt parieto-occipital & 6.0 & 1.54 & Intermediate & $>40$ to $<70$ \\
\hline 68 & 48 & $\mathrm{~F}$ & Rt clinoidal & 2.0 & 1.50 & Intermediate & $>40$ to $<70$ \\
\hline 69 & 48 & $\mathrm{M}$ & Tuberculum sella & 3.2 & 1.52 & Intermediate & $>40$ to $<70$ \\
\hline 70 & 87 & M & Rt parasagittal & 3.4 & 1.12 & Intermediate & 60 \\
\hline 71 & 45 & $\mathrm{~F}$ & Rt parasagittal & 4.9 & 1.65 & Intermediate & $>40$ to $<70$ \\
\hline 72 & 29 & $\mathrm{M}$ & Rt petrosal & 5.4 & 1.58 & Intermediate & $>40$ to $<70$ \\
\hline 73 & 82 & $\mathrm{~F}$ & Lt parasagittal & 5.1 & 2.22 & Intermediate & $>40$ to $<70$ \\
\hline 74 & 28 & $\mathrm{M}$ & Rt falcine & 1.8 & 1.08 & Intermediate & $>40$ to $<70$ \\
\hline 75 & 37 & $\mathrm{~F}$ & Rt clinoid & 3.6 & 1.58 & Intermediate & $>40$ to $<70$ \\
\hline 76 & 74 & $M$ & Rt CPA & 3.4 & 1.61 & Intermediate & $>40$ to $<70$ \\
\hline 77 & 61 & $\mathrm{~F}$ & Rt temporal & 3.7 & 1.42 & Intermediate & $>40$ to $<70$ \\
\hline 78 & 40 & $\mathrm{~F}$ & Rt jugular foramen & 4.7 & 1.30 & Intermediate & $>40$ to $<70$ \\
\hline 79 & 59 & $\mathrm{~F}$ & Lt tentorium & 3.0 & 1.01 & Intermediate & 50 \\
\hline 80 & 36 & $\mathrm{~F}$ & Foramen magnum & 2.5 & 0.88 & Firm & $70-100$ \\
\hline 81 & 46 & $\mathrm{~F}$ & Lt petrous ridge & 3.7 & 0.42 & Firm & $70-100$ \\
\hline 82 & 52 & $\mathrm{~F}$ & Pineal region & 2.1 & 0.71 & Firm & $70-100$ \\
\hline 83 & 51 & $\mathrm{~F}$ & Lt occipital convexity & 4.1 & 0.53 & Firm & $70-100$ \\
\hline 84 & 72 & $\mathrm{~F}$ & Rt sphenoid wing & 2.5 & 0.72 & Firm & 80 \\
\hline 85 & 64 & $\mathrm{~F}$ & Rt falcine & 6.0 & 1.50 & Firm & $70-100$ \\
\hline 86 & 82 & M & Rt frontal convexity & 2.4 & 0.94 & Firm & $70-100$ \\
\hline 87 & 65 & $\mathrm{~F}$ & Lt occipitoparietal & 8.6 & 0.88 & Firm & 100 \\
\hline 88 & 81 & $\mathrm{~F}$ & Lt falcine & 3.9 & 1.18 & Firm & 80 \\
\hline 89 & 67 & $\mathrm{~F}$ & Rt posterior fossa & 2.9 & 1.27 & Firm & 80 \\
\hline 90 & 56 & $M$ & Petroclival & 1.0 & 1.24 & Firm & $70-100$ \\
\hline 91 & 53 & $\mathrm{~F}$ & Rt cavernous & 2.0 & 0.86 & Firm & $70-100$ \\
\hline 92 & 74 & $\mathrm{~F}$ & Lt falcine & 3.6 & 1.19 & Firm & $70-100$ \\
\hline 93 & 70 & $\mathrm{~F}$ & Foramen magnum & 2.0 & 1.64 & Firm & 80 \\
\hline 94 & 52 & $\mathrm{~F}$ & Cervicothoracic & 2.2 & 0.67 & Firm & $70-100$ \\
\hline 95 & 61 & $\mathrm{~F}$ & Petroclival & 3.8 & 1.27 & Firm & $70-100$ \\
\hline 96 & 69 & $\mathrm{~F}$ & Lt tentorial & 8.0 & 0.34 & Firm & $70-100$ \\
\hline
\end{tabular}


» CONTINUED FROM PAGE 246

TABLE 2. Summary of data on 100 patients with meningioma

\begin{tabular}{rcclcccc}
\hline Case No. & Age $(\mathrm{yrs})$ & Sex & Tumor Location & Size $(\mathrm{cm})$ & TCTI Ratio & Clinical Consistency & CUSA Setting \\
\hline 97 & 50 & F & Sphenoid wing & 4.2 & 0.79 & Firm & $70-100$ \\
\hline 98 & 49 & F & Parasagittal & 6.0 & 1.06 & Firm & 100 \\
\hline 99 & 76 & F & Olfactory groove & 3.6 & 0.81 & Firm & 100 \\
\hline 100 & 83 & M & Lt sphenoid & 1.4 & 1.49 & Firm & $70-100$ \\
\hline
\end{tabular}

$\mathrm{CPA}=$ cerebellopontine angle.

that $\mathrm{T} 2$ hyperintensity relative to cortex probably indicated soft consistency and syncytial or angioblastic subtypes; however, in this study intensity was relative and not a numerical value. ${ }^{9}$ The majority of studies report no correlation between meningioma consistency and $\mathrm{T} 1$ intensity or gadolinium enhancement. ${ }^{13}$ Fractional anisotropy showed a statistically significant correlation but requires a highercomplexity imaging technique and is less generalizable. ${ }^{6}$ Additionally, MR elastography has begun to show promise in predicting tumor consistency but also requires special techniques. ${ }^{5,10}$

However, this correlation with imaging intensity has not been consistently demonstrated. Kasoff et al., Kashimura et al., and Carpeggiani et al. did not find any statistically significant correlation between meningioma consistency and T2 intensity. ${ }^{1,6,7}$ Proton density studies have shown conflicting results, with some showing a statistically significant correlation, while others lacked significant results. ${ }^{1,16,19}$

As no standard prediction method exists, we experimented with multiple MRI sequences to find one that correlated with objective consistency grades and required no special equipment or interpretation. The T2-weighted MRI sequence showed the highest degree of correlation in many studies discussed above and in our own analysis. ${ }^{3,4,9,13,16,19,20}$ In another study T2 FLAIR imaging showed a potential correlation, ${ }^{13}$ but in the present study it failed to show a correlation with our ratio method. Given the nonuniformity in MR scanners, it is possible that discrepancies in previous T2-weighted series are a result of various scanners and protocols. Thus we used the intensity ratio to standardize the intensity values with an internal reference.

Our secondary goal was to create an objective scale for rating clinical tumor consistency intraoperatively. Many current methods rely on subjective surgeon ranking or the use of various instruments. However, these systems are subjective and somewhat categorical, not allowing for con-

TABLE 3. Tumor consistency group statistics

\begin{tabular}{lcccc}
\hline & & \multicolumn{3}{c}{ Tumor Consistency Grade } \\
\cline { 3 - 5 } Category & Overall TCTI Ratio & $\mathrm{A}$ & $\mathrm{B}$ & $\mathrm{C}$ \\
\hline Mean & 1.59 & 1.91 & 1.49 & 1.01 \\
\hline Median & 1.60 & 1.84 & 1.50 & 0.88 \\
\hline Minimum & 0.34 & 1.63 & 1.33 & 0.34 \\
\hline Maximum & 3.79 & 3.79 & 1.63 & 1.27 \\
\hline SD & & 0.514 & 0.289 & 0.419 \\
\hline $95 \% \mathrm{Cl}$ & & $1.76-2.05$ & $1.38-1.60$ & $0.82-1.20$ \\
\hline
\end{tabular}

sistency grading along the true continuum of meningiomas. Using a scale that incorporates the minimum CUSA amplitude places clinical tumor consistency on a numeric continuum. This objectivity allows generalization of the TCTI ratios to other surgeons and is one way in which our technique is unique and complements previous attempts to quantify consistency. The main limitation of this rating method is the availability of CUSA equipment.

In our study 100 consecutive patients were analyzed for imaging characteristics and intraoperative tumor consistency. We used TCTI ratios to improve the objectivity and generalizability of this numerical ranking. In addition, by providing an internal reference (cerebellar peduncle), we reduced scanner variability.

Tumor consistency correlated with T2-weighted imaging characteristics (TCTI ratios). Softer tumors correlated with ratios $>1.63$ (greater intensity of tumor) and were removed with suction alone or a CUSA amplitude $<40$. Firm tumors were correlated with ratios $<1.27$ and necessitated a CUSA amplitude $>70$ or surgical adjuncts. In comparing consistency extremes, a TCTI of 1.41 was $81.9 \%$ sensitive and $84.8 \%$ specific for soft $(\geq 1.41)$ versus firm $(<1.41)$ tumors. Preoperative prediction of meningioma consistency adds value for surgical planning and preparation.

\section{Conclusions}

The consistency of meningiomas is an important aspect of surgical planning and technique. As tumor consistency

TABLE 4. Summary of imaging-consistency correlation studies

\begin{tabular}{lrrrrrrr}
\hline & No. of & \multicolumn{5}{c}{ Correlation With Consistency } \\
\cline { 3 - 8 } \multicolumn{1}{c}{ Authors \& Year } & Patients & T1 & T2 & PD & FLAIR & FA \\
\hline Chen et al., 1992 & 54 & - & + & NS & NS & NS \\
\hline Zee et al., 1992 & 54 & - & + & NS & NS & NS \\
\hline Carpeggiani et al., 1993 & 43 & - & - & - & NS & NS \\
\hline Suzuki et al., 1994 & 73 & - & + & + & NS & NS \\
\hline Kasoff et al., 1995 & 67 & - & - & NS & NS & NS \\
\hline Yamaguchi et al., 1997 & 50 & - & + & + & NS & NS \\
\hline Maiuri et al., 1999 & 35 & - & + & NS & NS & NS \\
\hline Kashimura et al., 2007 & 29 & NS & - & NS & NS & + \\
\hline Hoover et al., 2011 & 101 & - & + & NS & NS & NS \\
\hline Sitthinamsuwan et al., 2012 & 243 & - & + & NS & + & NS \\
\hline Watanabe et al., 2015 & 43 & - & + & - & + & NS \\
\hline Present study & 100 & - & + & - & - & NS \\
\hline
\end{tabular}

* FA = fractional anisotropy; NS = not studied; + = positive correlation with consistency; - = no correlation with consistency. 
follows a histological continuum, predicting extremes such as soft (easily resectable) or firm (hard to resect) is perhaps the most clinically relevant option at a cutoff TCTI ratio of 1.41. This intensity ratio showed good prediction sensitivity and specificity, with a positive predictive value of $84.9 \%$ and positive likelihood ratio of 5.39. Overall, meningioma consistency compared with tumor MRI intensity, internally referenced to the MRI intensity of the cerebellar peduncle (TCTI ratio), showed good correlation and, we hope, will improve preoperative planning for meningioma surgery.

\section{References}

1. Carpeggiani P, Crisi G, Trevisan C: MRI of intracranial meningiomas: correlations with histology and physical consistency. Neuroradiology 35:532-536, 1993

2. Chen TC, Zee CS, Miller CA, Weiss MH, Tang G, Chin L, et al: Magnetic resonance imaging and pathological correlates of meningiomas. Neurosurgery 31:1015-1022, 1992

3. Chernov MF, Kasuya H, Nakaya K, Kato K, Ono Y, Yoshida $\mathrm{S}$, et al: ${ }^{1} \mathrm{H}-\mathrm{MRS}$ of intracranial meningiomas: what it can add to known clinical and MRI predictors of the histopathological and biological characteristics of the tumor? Clin Neurol Neurosurg 113:202-212, 2011

4. Hoover JM, Morris JM, Meyer FB: Use of preoperative magnetic resonance imaging $\mathrm{T} 1$ and $\mathrm{T} 2$ sequences to determine intraoperative meningioma consistency. Surg Neurol Int 2:142, 2011

5. Hughes JD, Fattahi N, Van Gompel J, Arani A, Meyer F, Lanzino G, et al: Higher-resolution magnetic resonance elastography in meningiomas to determine intratumoral consistency. Neurosurgery 77:653-659, 2015

6. Kashimura H, Inoue T, Ogasawara K, Arai H, Otawara Y, Kanbara Y, et al: Prediction of meningioma consistency using fractional anisotropy value measured by magnetic resonance imaging. J Neurosurg 107:784-787, 2007

7. Kasoff SS, Spiller M, Valsamis MP, Lansen TA, Duffy KR, Koenig SH, et al: Relaxometry of noncalcified human meningiomas. Correlation with histology and solids content. Invest Radiol 30:49-55, 1995

8. Madabhushi A, Udupa JK, Moonis G: Comparing MR image intensity standardization against tissue characterizability of magnetization transfer ratio imaging. J Magn Reson Imaging 24:667-675, 2006

9. Maiuri F, Iaconetta G, de Divitiis O, Cirillo S, Di Salle F, De Caro ML: Intracranial meningiomas: correlations between MR imaging and histology. Eur J Radiol 31:69-75, 1999

10. Murphy MC, Huston J III, Glaser KJ, Manduca A, Meyer FB, Lanzino G, et al: Preoperative assessment of meningioma stiffness using magnetic resonance elastography. J Neurosurg 118:643-648, 2013

11. Pfisterer WK, Nieman RA, Scheck AC, Coons SW, Spetzler $\mathrm{RF}$, Preul MC: Using ex vivo proton magnetic resonance spectroscopy to reveal associations between biochemical and biological features of meningiomas. Neurosurg Focus 28(1):E12, 2010
12. Robitaille N, Mouiha A, Crepeault B, Valdivia F, Duchesne S: The Alzheimer's Disease Neuroimaging I: Tissue-based MRI intensity standardization: application to multicentric datasets. Int J Biomed Imaging 2012:347120, 2012

13. Sitthinamsuwan B, Khampalikit I, Nunta-aree S, Srirabheebhat P, Witthiwej T, Nitising A: Predictors of meningioma consistency: A study in 243 consecutive cases. Acta Neurochir (Wien) 154:1383-1389, 2012

14. Smith KA, Leever JD, Chamoun RB: Predicting consistency of meningioma by magnetic resonance imaging. $\mathbf{J}$ Neurol Surg B Skull Base 76:225-229, 2015

15. Soyama N, Kuratsu J, Ushio Y: Correlation between magnetic resonance images and histology in meningiomas: T2-weighted images indicate collagen contents in tissues. Neurol Med Chir (Tokyo) 35:438-441, 1995

16. Suzuki Y, Sugimoto T, Shibuya M, Sugita K, Patel SJ: Meningiomas: correlation between MRI characteristics and operative findings including consistency. Acta Neurochir (Wien) 129:39-46, 1994

17. Wang D, Doddrell DM: Method for a detailed measurement of image intensity nonuniformity in magnetic resonance imaging. Med Phys 32:952-960, 2005

18. Watanabe K, Kakeda S, Yamamoto J, Ide S, Ohnari N, Nishizawa S, et al: Prediction of hard meningiomas: quantitative evaluation based on the magnetic resonance signal intensity. Acta Radiol 57:333-340, 2016

19. Yamaguchi N, Kawase T, Sagoh M, Ohira T, Shiga H, Toya S: Prediction of consistency of meningiomas with preoperative magnetic resonance imaging. Surg Neurol 48:579-583, 1997

20. Yrjänä SK, Tuominen H, Karttunen A, Lähdesluoma N, Heikkinen E, Koivukangas J: Low-field MR imaging of meningiomas including dynamic contrast enhancement study: evaluation of surgical and histopathologic characteristics. AJNR Am J Neuroradiol 27:2128-2134, 2006

21. Zee CS, Chen T, Hinton DR, Tan M, Segall HD, Apuzzo ML: Magnetic resonance imaging of cystic meningiomas and its surgical implications. Neurosurgery 36:482-488, 1995

\section{Disclosures}

The authors report no conflict of interest concerning the materials or methods used in this study or the findings specified in this paper.

\section{Author Contributions}

Conception and design: Chamoun. Acquisition of data: all authors. Analysis and interpretation of data: Smith. Drafting the article: Smith. Critically revising the article: Chamoun, Smith. Reviewed submitted version of manuscript: Chamoun, Smith. Approved the final version of the manuscript on behalf of all authors: Chamoun.

\section{Correspondence}

Roukoz Chamoun, University of Kansas Medical Center, 3901 Rainbow Blvd., Mailstop 3021, Kansas City, KS 66160. email: rchamoun@kumc.edu. 